\title{
Influencia de curso precálculo y actividades de apoyo institucional en desarrollo de competencias y creencias en matemáticas
}

\section{Influence of pre-calculus course and institutional support activities in the development of competences and beliefs in mathematics}

\section{Influencia de curso pré-cálculo e atividades de apoio institucional no desenvolvimento de competências e crenças em matemáticas}

\author{
Mawency Vergel-Ortega ${ }^{1}$, Rosa Virginia Hernández², Olga Lucy Rincón-Leal ${ }^{3}$
}

Forma de citar: M. Vergel-Ortega, R. V. Hernández, O. L. Rincón-Leal, "Influencia de curso precálculo y actividades de apoyo institucional en desarrollo de competencias y creencias en matemáticas", Eco.Mat. vol. 7, no. 1, pp. 33-47, 2016

Recibido:

Julio 14 de 2015

Aceptado:

Diciembre 6 de 2015

\section{Resumen}

La investigación siguió un enfoque cuantitativo con apoyo del enfoque cualitativo, descriptivo, diseño cuasi experimental. En esta investigación se plantearon los objetivos: estudiar si mejoran las competencias en pensamiento variacional y didácticas de los estudiantes con la utilización de curso precálculo utilizando plataforma virtual respecto a enseñanza tradicional; examinar la influencia en las creencias sobre las matemáticas y su enseñanza; así como analizar qué tipología de alumnos obtiene mejores resultados en relación a su nivel de competencia digital. Se han obtenido las siguientes conclusiones: el grupo experimental ha obtenido una mejora estadísticamente significativa de sus competencias interpretativas y de pensamiento variacional respecto al grupo control, las creencias sobre las matemáticas mejoran en ambos grupos del postest al pretest, pero no puede atribuirse al uso de herramientas web.

${ }^{1}$ Doctora en Educación mawency@ufps.edu.co Universidad Francisco de Paula Santander Cúcuta- Colombia

${ }^{2}$ Magister en Educación Matemática rosavirginia@ufps.edu.co, Universidad Francisco de Paula Santander

Cúcuta- Colombia

${ }^{3}$ Magister en Educación Matemática

olgarincon@ufps.edu.co ORCID: 0000-0002-8080-496X Universidad Francisco de Paula Santander Cúcuta- Colombia
Palabras clave: competencias creencias sobre las matemáticas, precálculo, software.

\begin{abstract}
The research followed a quantitative approach with the support of qualitative, descriptive, semi-experimental design. The objectives of this research were: to study if competences in variation and didactic thinking of the students improve when taking a pre-calculus course using virtual platform compared to traditional teaching; examine the influence on beliefs about mathematics and its teaching; as well as to analyze which typology of students obtains better results in relation to their level of digital competence. The following conclusions have been drawn: the experimental group has achieved a statistically significant improvement of its interpretive and variation thinking skills compared to the control group; beliefs about mathematics improve in both groups from post-test to pretest, but it cannot be attributed to the use of Web tools.
\end{abstract}

Keywords: beliefs about mathematics, software, pre-calculus, competences. 
No. 1

Enero-Diciembre 2016 ISSN 1794-8231 E-ISSN $2462-8794$ PP: $33-47$

\begin{abstract}
Resumo
A pesquisa seguiu um enfoque quantitativo com apoio do enfoque qualitativo, descritivo e delineamento quase experimental. Nesta pesquisa se plantearam os objetivos: estudar se as competências em pensamento variacional e didáticas dos estudantes melhoram com a utilização de um curso pré-cálculo empregando plataforma virtual respeito a ensino tradicional; examinar a influência nas crenças sobre as matemáticas e seu ensino; assim como analisar que tipologia de alunos obtém melhores resultados em relação a seu nível de competência digital. Obtiveram-se as seguintes conclusões: o grupo experimental obteve uma melhora estatisticamente significativa de suas competências interpretativas e de pensamento variacional respeito ao grupo controle, as crenças sobre as matemáticas melhoraram em ambos os grupos do pós-teste ao pré-teste, mas não conseguiram ser atribuídas ao uso de ferramentas da web.
\end{abstract}

Palavras-chave: crenças sobre as matemáticas, software, pré-cálculo, competências.

\section{Introducción}

Mejorar la capacidad institucional a través del desarrollo de estrategias y metodologías que garanticen la permanencia y graduación estudiantil contribuyendo con el proceso de calidad académica en la universidad. Cálculo, matemática I es una disciplina más importantes de las matemáticas, debido a su aplicación en todas las ciencias y áreas, que gran parte del desarrollo tecnológico del siglo XX descansa sobre su campo de aplicación, por lo tanto es un área obligatoria en el estudio las Ciencias Exactas, Sociales, y carreras de ciencias básicas.

A pesar de que dentro del sistema escolar, el cálculo ocupa un lugar privilegiado, en el sentido de que antes de él está la "matemática elemental"; y después de él inicia la matemática avanzada, y es aquí donde se empieza con las dificultades y complejidades de un relativamente nuevo campo de estudio [1].

Los problemas de enseñanza-aprendizaje de Cálculo, son persistentes por lo que es alarmante la deserción. Como respuesta a esta problemática han surgido diversos trabajos de investigación en el campo de la
Matemática Educativa [2], [3], [4], [5] y [6]. La presente propuesta de investigación consiste en Estimar un modelo estructural que posibilite las relaciones causales y no causales que midan el constructo plataforma virtual en la enseñanza del cálculo.

De otra parte, la globalización que se vive actualmente obliga a los países a desarrollara su talento humano para garantizar una economía floreciente y un nivel de competitividad básico por lo que la atención a jóvenes con aptitudes sobresalientes resulta primordial; asimismo se debe capacitar al docente para la atención de este tipo de alumnos. Muchos estudios se han realizado en torno a cómo mejorar el bajo rendimiento en las matemáticas; durante 2005, Institutos con experiencias significativas en implementación de tics como el instituto Oficial Mixto Leónidas Méncos Avila, de Tiquisate, se precisó que un factor determinante para el bajo rendimiento académico en el área de matemáticas estaba dado por la metodología empleada por el docente, que no era motivadora [7]. Las universidades han implementado plataformas observando la necesidad de construcción de herramientas didácticas que superen los métodos tradicionales de enseñanza, dándoles a los jóvenes una formación en diferentes 
contextos. Situación que superaría tendencias a centrarse en una práctica algorítmica y algebraica que terminan siendo rutinarias. Como lo reporta en las investigaciones en Francia [8] "los estudiantes tienden a realizar procedimientos algorítmicos, sin tener en cuenta los conceptuales".

Sin embargo, no ignorando lo anterior, no se puede reducir que la reprobación de los cursos de cálculo e introducción a la vida universitaria, se deba a problemas de otras ramas de la matemática como el álgebra y procesos de selección de carrera en los estudiantes.

Las políticas gubernamentales en Colombia, han conllevado al establecimiento de políticas internas institucionales que refleja, la voluntad política del gobierno y de las universidades para construir e identificar factores que mejoren la deserción, es decir permitan la permanencia estudiantil, la graduación, mejoren el sentido de pertenencia institucional, se apropien de su misión, visión, reglamentos y elementos corporativos. No obstante, este proceso de construcción apenas comienza, lo cual requiere de acciones que permitan el compromiso de todos los actores que vivirán la transición de un país en guerra, desigual socialmente, a un país que se apropia de un conjunto de valores, actitudes y comportamientos que reflejan la docencia, investigación, extensión y la innovación.

Los modelos implementados en Colombia, han dado la posibilidad de cambiar las percepciones de algunos actores de la sociedad civil, que permiten la apropiación de conocimiento, camino deseable que tiene en cuenta factores estructurales, que pueden contribuir a reducir la deserción en cálculo o de programas académicos [9]. De esta manera, se requiere de una visión multicausal, jerárquica, de análisis factorial confirmatorio, que de forma objetiva, generen un marco de actuación, para consolidar los procesos y planes que han permitido la construcción y apropiación de la paz en los actores inmersos en la violencia, de manera que se evite causas que promueven la deserción estudiantil.

Ahondando aún más allá de opiniones o argumentaciones, como aquellas si el estudiante no sabe bien sumar, multiplicar o desarrollar ejercicios de álgebra como realmente lo conciben la mayoría de los cursos actuales tradicionales de Cálculo, podemos decir que la razón de su fracaso en la enseñanza en de éste, se polariza en dos extremos, que son:

- La fuerte carga operativa, la cual causa un deterioro conceptual [2]

- La enseñanza del Cálculo ejercida con una fuerte herencia de la Matemática formal [2] y [3]

La primera causa se refiere, a que los estudiantes mecanizan y aprenden de memoria un conjunto de fórmulas, técnicas, y definiciones de forma objetivizada, según [10] de manera ya elaborada en la cual el estudiante no interioriza o entiende a fondo el por qué y qué son las cosas, llevando finalmente a la creencia de que hacer matemáticas es realizar operaciones puntuales, manipular signos y memorizar [11] y [12].

Respecto a la segunda problemática [3] que menciona sobre la enseñanza del Cálculo con gran formalidad, se basan [13] argumentando que como consecuencia del tratado del Cálculo por los matemáticos del siglo XIX, en la actualidad se preocupan más por la formalización, en vez del desarrollo de métodos genuinos y el desarrollo de problemas basados en las ideas centrales que son la acumulación y variación, como los problemas desarrollados por Fermat, Cavalieri, Descartes, Wallis, entre otros... [14], antes de que se reconociera el Cálculo como otra rama más de la Matemática.
Enero-Diciembre 2016 ISSN 1794-8231 E-ISSN 2462 - 8794 PP: 33-47 
En el mismo sentido, [15] argumenta que existe una dislexia escolar en Cálculo, en la que su enseñanza logra que los estudiantes deriven y calculen límites elementales, pero no son capaces de dar un sentido más amplio a esas nociones que les haga reconocer, por ejemplo, cuándo un problema requiere de calcular una derivada; lo cual indica que la enseñanza del Cálculo está concentrada en el desarrollo operativo, sin reflexionar en su importancia aplicativa en problemas contextuales.

\section{Metodología}

La investigación sigue un enfoque cuantitativo, diseño explicativo, de tipo campo descriptivo con apoyo del análisis cualitativo desde una perspectiva interpretativa en la que se tuvieron en cuenta diversos aspectos cognitivos y procedimentales. Los estudios descriptivos buscan especificar las propiedades importantes de personas, grupos, comunidades o cualquier otro fenómeno que sea sometido a análisis" [16]. El diseño de la investigación es de naturaleza transversal, en el cual participaron 1085 estudiantes que cursaron en el segundo semestre del 2009 con edades comprendidas entre 18 y 30 años.

\section{Fase de Recolección de Información}

El plan seguido para la consecución de los objetivos es el siguiente: Revisión de Literatura.
Definición del objeto de estudio. Aplicación de pre-test, Diseño y validez de instrumentos Prueba piloto y validación de resultados, Rediseño de actividades, herramientas, contenido y su respectivo trabajo de campo, Recolección de Información, Confirmatorio de resultados:

En esta etapa, una vez que se dispone de los datos, estos se transforman y estudian para obtener los resultados que permitan concluir sobre la investigación. Se emplearan métodos estadísticos y se representaran en tablas y/o gráficos los resultados obtenidos. Formulación de conclusiones y definición de líneas futuras de investigación, instrumento para la recolección de información, se utilizaran cuestionarios, instrumentos de medición (Pre-test. y Post- test), Análisis cualitativo. La teoría de la instrumentación [17] ha servido de referente para el análisis cualitativo realizado en estudio de casos. El análisis se realizó bajo una perspectiva interpretativa en la que se tuvieron en cuenta diversos aspectos cognitivos y procedimentales

El presente artículo muestra resultados de investigación adelantada con los estudiantes matriculados para el segundo semestre académico del 2009 en los diferentes programas que ofrece la universidad Francisco de Paula Santander. De la totalidad de estudiantes matriculados (2009) en segundo semestre, se aplica el pretest a 1085 estudiantes de los diferentes programas ofertados por la Universidad Francisco de Paula Santander, excepto estudiantes de programas de Enfermería, Comunicación social y Arquitectura. El test se aplica en la segunda y tercer semana de clases.

El test aplicado está compuesto por 29 ítems con única respuesta, que abordan conceptos matemáticos básicos de operaciones con números racionales, simplificación de expresiones algebraicas, despeje de ecuaciones lineales, solución de ecuaciones cuadráticas, inecuaciones, trigonometría, plano cartesiano, geometría analítica y problemas de aplicación (3), cuya solución requería de la aplicación de regla de tres simple, planteamiento y solución de ecuación lineal y pendiente de una recta y modulo adicional de saber en contexto que incluyó 16 ítems de aplicación de matemática básica. La confiabilidad el test fue de 0.89 e índice de Kappa de 0.98. Incluyó ítems como: (Ver figura 1a, 1b, 1c) [18] 
Estimado estudiante, la presente evaluación constituye una prueba de sus conocimientos adquiridos durante la educación secundaria. Se desea contar con un diagnóstico para elaborar un diseño curricular que le permita a usted superar dificultades, si las tiene.

Por favor leer detenidamente las siguientes instrucciones:

- No escribir en el cuadernillo y regréselo al profesor al terminar.

- La prueba debe desarrollarse en un tiempo máximo de dos horas sin usar calculadora.

- La prueba debe ser diligenciada de forma individual.

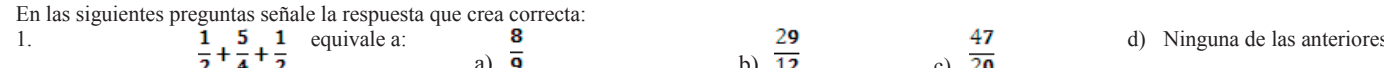

2. $\frac{3}{4} \div \frac{2}{3}$

$\frac{1}{2} \times \frac{4}{3} \times 5$

4. $1 \frac{3}{4}$

5. $\frac{x+2}{2 x+2}$

6. $\sqrt{x^{2}+4}$

7. $(2 x-1)^{2}$

8. $9 x^{2}-4$

9. $5 x+4=3 y$ equivale a:

equivale a:

equivale a:

equivale a:

equivale a:

equivale a:

equivale a:

equivale a: a) $\frac{1}{2}$

a) $\frac{10}{3}$

a) $\frac{13}{4}$

a) $\frac{1}{2}$

a) 2

a) $4 x^{2}-4 x+1$

a) $(3 x+2)(3 x-2)$

a) $y=5 x-1$ b) $\frac{29}{12}$

$\frac{17}{12}$

b) $\overline{12}$

b) 6

$\begin{array}{ll}\frac{3}{4} & \text { c) } \frac{7}{4}\end{array}$

b) $x+2$

b) $\sqrt{x+2}$

c) $\frac{x+2}{x}$

$\underline{9}$

c) $\overline{8}$

20

$\frac{7}{4}$

c) $x+2$

b) $\begin{array}{ll}4 x^{2}-1 & \text { c) } x^{2}\end{array}$

b) $(3 x-2)^{2} \quad$ c) $5 x^{2}$

b) $y=\frac{5 x+4}{3}$
PP: $33-47$

Figura 1a. Test de conocimientos adquiridos durante la educación secundaria Fuente [18]

\begin{tabular}{|c|c|c|c|c|c|c|c|c|c|c|}
\hline 10. & \multicolumn{2}{|c|}{ Las raíces de $x^{2}-x-2=0$ son: } & $\alpha)$ & $x=1, x=2$ & $\beta)$ & $x=-1, x=2$ & $\chi)$ & $x=2$ & ठ) & Ninguna de las anteriores \\
\hline 11. & $\frac{1}{2}\left[(a+b)^{2}-\left(a^{2}+b^{2}\right)\right]$ & equivale a: & a) & $\mathbf{0}$ & b) & $a b$ & c) & $b^{2}$ & d) & Ninguna de las anteriores \\
\hline 12. & \multicolumn{2}{|c|}{$\begin{array}{l}\text { La solución } a \frac{x^{2}+4 x-12}{x-2} \geq 0 \text { es } \\
\text { : }\end{array}$} & a) & {$[-6,0)$} & b) & {$[-6,2]$} & c) & $(-6,2)$ & d) & Ninguna de las anteriores \\
\hline 13. & $\sqrt{5-9}$ & equivale a: & a) & 2 & b) & $2 i$ & c) & -2 & d) & Ninguna de las anteriores \\
\hline 14. & $\frac{x^{2} y}{\sqrt{x}}$ & equivale a: & a) & $x^{\frac{3}{2}} y$ & b) & $x y$ & c) & $\sqrt{x y}$ & d) & Ninguna de las anteriores \\
\hline 15. & $\log (5)+\log (3)$ & equivale a: & a) & $\log (15)$ & b) & $\log (8)$ & c) & $\log \left(\frac{5}{3}\right)$ & d) & Ninguna de las anteriores \\
\hline 16. & $\log (6)-\log (2)$ & equivale a: & a) & $\log (3)$ & b) & $\log (4)$ & c) & $\log (12)$ & d) & Ninguna de las anteriores \\
\hline & $2 \log (\sqrt{7})$ & equivale a: & a) & $\sqrt{2 \log (7)}$ & b) & $\log \left(\frac{\sqrt{7}}{2}\right)$ & c) & $\log (7)$ & d) & Ninguna de las anteriores \\
\hline 18. & $15^{\circ}$ equivale en radia & es $a$ : & a) & $\frac{3 \pi}{2}$ & b) & $\frac{\pi}{12}$ & c) & $\frac{\pi}{8}$ & d) & Ninguna de las anteriores \\
\hline & $\cot (\alpha)$ se define cómo & & a) & $\frac{\cos (\alpha)}{\operatorname{sen}(\alpha)}$ & b) & $\frac{\operatorname{sen}(\alpha)}{\cos (\alpha)}$ & c) & $\cos ^{2}(\alpha)$ & d) & Ninguna de las anteriores \\
\hline
\end{tabular}

22. Las coordenadas de un punto que se halla sobre el eje " $y$ " a una altura desde el origen igual a 2 unidades, están dadas por:
a) $y=2$
b) $(0,2)$
c) $(2,0)$
d) Ninguna de las anteriores

Figura $1 \mathrm{~b}$. Test de conocimientos adquiridos durante la educación secundaria Fuente [18] 
23. La distancia existente entre los puntos $\mathrm{A}(1,2)$ y $\mathrm{B}(4,6)$ es:
a) 7 unidades
b) 3 unidades
c) 5 unidades
d) Ninguna de las anteriores

24. Un segmento de recta tiene un extremo en el origen y el otro extremo en el punto $\mathrm{C}(2,4)$. El punto medio del segmento está en:
a) $(1,2)$
b) $(3,3)$
c) $(0,3)$
d) Ninguna de las anteriores

25. Si la recta $\mathrm{L}_{1}$ está dada por la ecuación $\boldsymbol{y}=3 \boldsymbol{x}+4$ y la recta $\mathrm{L}_{2}$ está dada por la ecuación $\boldsymbol{y}=\mathbf{5} \boldsymbol{x}+\boldsymbol{1}$ entonces:

a) $\mathrm{L}_{1}$ está más inclinada que $\mathrm{L}_{2} \quad$ b) $\mathrm{L}_{2}$ está más inclinada que $\mathrm{L}_{1} \quad$ c) $\mathrm{L}_{1}$ y $\mathrm{L}_{2}$ tienen la misma inclinación d) Ninguna de las anteriores

26. El punto de corte entre las rectas $\mathrm{L}_{1}: \boldsymbol{y}=\boldsymbol{x}$ y $\boldsymbol{L}_{2}: \boldsymbol{y}=\mathbf{2} \boldsymbol{x}+1$, está en:
a) No se cortan
b) $(2,1)$
c) $(-1,-1)$
d) Ninguna de las anteriores
27. $x^{2}+y^{2}=1$ representa una:
a) Parábola
b) Elipse
c) Hipérbola
d) Ninguna de las anteriores
28. $y=x^{2}$ representa una:
a) Circunferencia
b) Elipse
c) Parábola
d) Ninguna de las anteriores
29. $x^{2}-y^{2}=1$ representa una:
a) Circunferencia
b) Elipse
c) Hipérbola
d) Ninguna de las anteriores
30. De los $\$ 50.000$ que tenía Priscila, gastó el $25 \%$ en el almuerzo, compró una blusa con el $40 \%$ y en transporte utilizó el $20 \%$. ¿Cuánto dinero le queda a Priscila? Respuesta:
31. Una tabla de $180 \mathrm{~cm}$ debe ser cortada en tres partes, de tal manera que la segunda parte sea $10 \mathrm{~cm}$ más larga que la primera y que la tercera sea $10 \mathrm{~cm}$ más largo que la segunda. ¿De qué longitud debe ser cada una de las partes? Respuesta:
32.
1
Una vela mide 6 pulgadas después de estar encendida una hora. Después de 3 horas, mide $5 \overline{2}$ pulgadas. La ecuación que determina la altura de la vela dónde la pendiente es

Figura 1c. Test de conocimientos adquiridos durante la educación secundaria Fuente [18]

\subsection{Programas de apoyo a la implementación}

\subsubsection{Sensibilización y posicionamiento del Tema.}

Durante los años 2007 a 2010 se realizaron pruebas previas de precálculo y un módulo virtual de enseñanza previa de uso gratuito para estudiantes de pregrado y estudiantes de educación media. Durante los años 2011 a 2014, de acuerdo a plan de desarrollo institucional 2011-2019, y de acuerdo a análisis longitudinal de factores que inciden en la deserción de estudiantes de la Universidad Francisco de Paula Santander [19] y [20], se diseñó y propuso ante entes académicos una política para la gestión de la permanencia y graduación estudiantil en la Universidad Francisco de Paula Santander. Acompañando la propuesta a través del programa Quédate [21] con jornadas de sensibilización y socialización en los diferentes estamentos; estudiantes, directivos, docentes y funcionarios administrativos, así mismo se implementaron acciones como aplicación de pretest para analizar preconceptos en cálculo en estudiantes de grado 11 de educación media y en estudiantes que ingresaban por primera vez a la universidad, curso de precálculo para en estudiantes de primer semestre de la asignatura introducción a la vida universitaria. El programa incluyó la vinculación de padres de familia en el proceso, concursos en geometría, olimpiada matemática universitaria y para educación básica y media; acompañamiento psicológico y pedagógico a estudiantes, gestión de monitorias personalizadas y grupales, apoyo a través de asesoría virtual y ampliación de cupos en comedor estudiantil dado el nivel socioeconómico de los estudiantes y jornadas de intervención de resultados, políticas, proyectos y estímulos a cada programa académico.

\subsubsection{Cultura de la información y de la evaluación de resultados}

A través de jornadas de Capacitación en 
la herramienta SPADIES, a través de la herramienta se realizó diagnóstico de riesgo alto, moderado y bajo en deserción, por lo anterior se implementaron estrategias de intervención y permanencia. Publicación en la web de bienestar universitario de los resultados y avances.

\subsubsection{Mejoramiento de la calidad académica y articulación entre niveles}

Orientar a los docentes en políticas y estrategias metodológicas para fomentar la permanencia y graduación estudiantil. [22], Formar a docentes en competencias cognitivas, desarrollo del aprendizaje y factores psicosociales [23] que fomente el aprendizaje de los estudiantes.

Capacitación a los estudiantes beneficiados en el acuerdo 012, Seguimiento académico y psicosocial a los estudiantes que se beneficiaron del Acuerdo 012

\subsubsection{Gestión de recursos}

Contribuir a los estudiantes que se encuentren en situación precaria y logren culminar con éxito su programa académico, Reliquidación del valor de matrícula [24], Becas Trabajo Servicio de Comedor.

\subsubsection{Trabajo colaborativo en red}

Diseño y elaboración de módulos: Orientación académica. Desarrollo de estrategias pedagógicas (enfoques de aprendizajes y motivación), Test de orientación vocacional Herramientas para un aprendizaje efectivo métodos técnicas y hábitos de estudio.

\subsubsection{Fortalecimiento del programa Quédate}

Realizar actividades en: Prevención y reducción del consumo de sustancias psicoactivas Talleres y Asesoría en
Orientación Académica y psicológica, Estilos de vida Saludable en desarrollo humano y salud integral

\subsubsection{Escuela de padres}

Realizar talleres psicoeducativos a padres de familia que conforman la escuela de padres. Herramientas para identificar si mi hijo es consumidor de SPA, Factores de crianza positiva

Comunicación Asertiva, Heridas emocionales, Métodos y hábitos de estudio, Prevención y reducción del consumo de sustancias psicoactivas, Adaptación a la vida Universitaria

\subsubsection{Teoría de orquestación instrumental}

Esta idea desarrollada por [25] es una forma que permite al profesor y estudiantes a desarrollar una Génesis Instrumental mediante un proceso de instrumentación, este proceso el cual consiste el convertir un artefacto en una herramienta y finalmente en un instrumento. Por ejemplo: un smarthphones es un artefacto para alguien que no le da uso, cuando este artefacto es utilizado para un fin en el que fue diseñado entonces se considera una herramienta. Cuando la herramienta es utilizada para fines que estén fuera de las funciones básicas, es decir al considerar el smarthphones más que un dispositivo para hacer llamadas, tomar fotos y utilizarlo como una herramienta cognitiva, podemos decir que es allí donde hay un apoderamiento de la herramienta la cual se considera un

\section{Resultados}

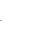

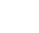


Enero-Diciembre 2016 ISSN 1794-8231 E-ISSN 2462 - 8794

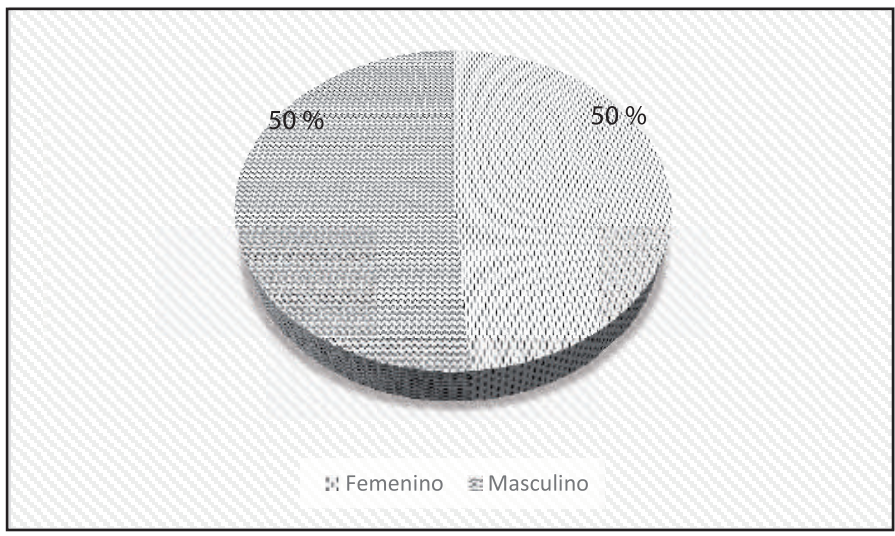

Figura 2. Estudiantes de primer semestre por género

Fuente: autores

En la tabla I se evidencia que la edad del $85 \%$ de los estudiantes que ingresaron a la universidad en el segundo semestre del 2009 oscila entre los 15 y 20 años.

Tabla I. Características estudiantes por edad en el primer semestre

\begin{tabular}{|c|c|c|}
\hline Edad & Frecuencia & Porcentaje \\
\hline De 15 a 17 & 385 & 35,5 \\
\hline De 18 a 20 & 540 & 49,8 \\
\hline De 21 a 23 & 106 & 9,8 \\
\hline De 24 a 26 & 23 & 2,1 \\
\hline De 27 a 29 & 18 & 1,6 \\
\hline Mayor a 30 & 13 & 1,2 \\
\hline TOTAL & $\mathbf{1 0 8 5}$ & $\mathbf{1 0 0}$ \\
\hline
\end{tabular}

Fuente: autores

Respecto al estrato socioeconómico, el 30.1\% de los estudiantes de primer semestre pertenece a Familias de estrato 1, un $53.4 \%$ a estrato 2, un $7.6 \%$ estrato 3, un 5.5\% a Estrato 4, 3.5\% a otros estratos (figura 3)

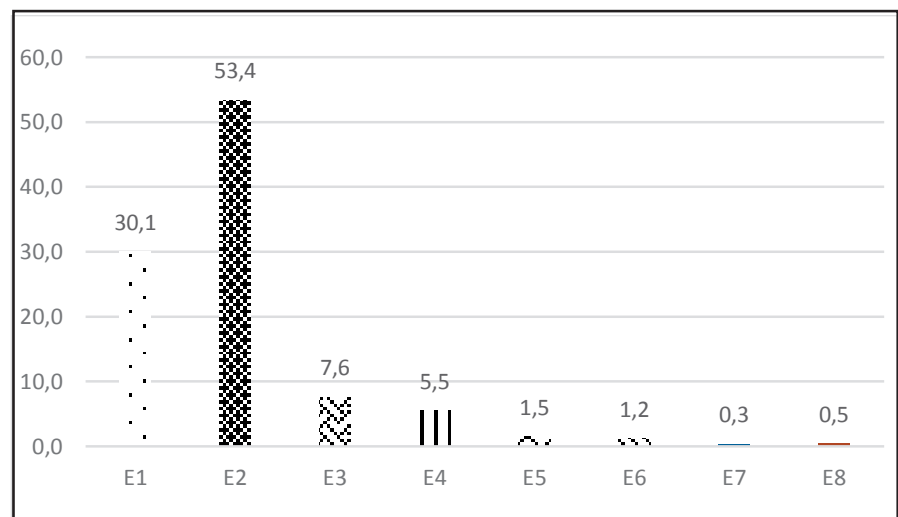

Figura 3. Estudiantes de primer semestre por estrato socioeconómico

Fuente: autores

E1 85\% de los estudiantes que ingresaron para el segundo semestre académico del 2009 iniciaron su acceso a la educación superior en éste período (figura 4). 


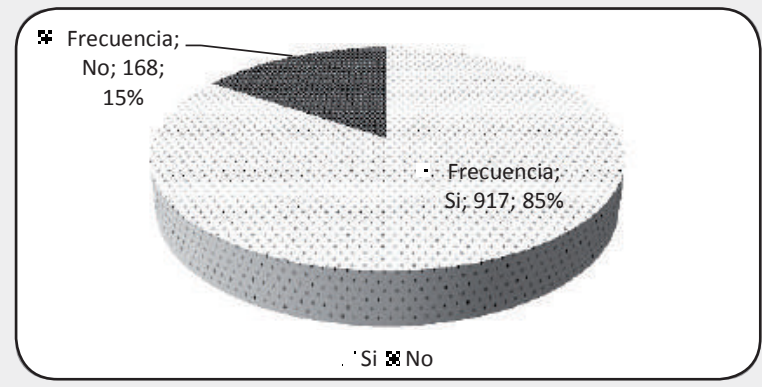

Enero-Diciembre 2016 ISSN 1794-8231

Figura 4. Estudiantes que ingresan por primera vez en una carrera de pregrado a la Universidad Fuente: autores

El 78\% de los estudiantes que ingresaron para el segundo semestre académico del 2009 terminaron sus estudios de bachillerato en instituciones de carácter público (figura 5).

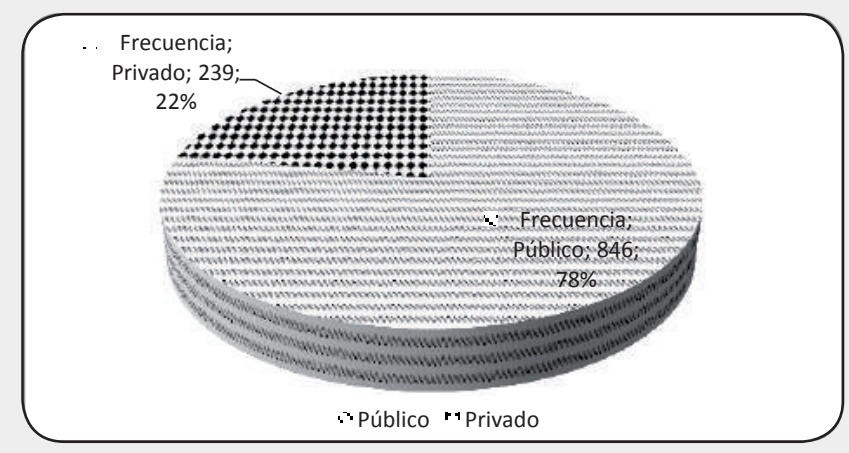

Figura 5. Tipo de Colegio que provienen Fuente: autores

La tabla II muestra que el $76 \%$ de los estudiantes terminaron sus estudios de bachillerato en un tiempo no mayor de dos años atrás.

Tabla II. Año de graduación
\begin{tabular}{|l|c|}
\hline \multicolumn{1}{|c|}{ Año de Egreso } & Porcentaje \\
\hline De 1990 a 2000 & $2,2 \%$ \\
\hline De 2001 a 2003 & $3,7 \%$ \\
\hline De 2004 a 2006 & $17,1 \%$ \\
\hline Año 2007 & $20,3 \%$ \\
\hline Año 2008 & $55,9 \%$ \\
\hline Año 2009 & $0,8 \%$ \\
\hline TOTAL & $\mathbf{1 0 0} \%$ \\
\hline
\end{tabular}

Fuente: autores

La tabla III muestra que un $21 \%$ de los estudiantes que ingresaron a primer semestre por primera opción, en el II semestre académico de 2009 corresponde al programa de Administración de Empresas. Se observa baja demanda en programas de Tecnología Química y Licenciaturas. 
No. 1

Enero-Diciembre 2016 ISSN 1794-8231 E-ISSN $2462-8794$ PP: $33-47$
Tabla III. Distribución por programa académico

\begin{tabular}{|l|c|}
\hline \multicolumn{1}{|c|}{ Programa Académico } & Porcentaje \\
\hline Ing. Agronómica & $5,1 \%$ \\
\hline Ing. Mecánica & $9,2 \%$ \\
\hline Ing. Civil & $4,4 \%$ \\
\hline Ing. de Sistemas & $5,1 \%$ \\
\hline Ing. Electrónica & $5,3 \%$ \\
\hline Ing. Electromecánica & $6,3 \%$ \\
\hline Ing. Industrial & $3,2 \%$ \\
\hline Administración de Empresas & $20,8 \%$ \\
\hline Contaduría Pública & $19,1 \%$ \\
\hline Lic. Biología-Química & $1,3 \%$ \\
\hline Lic. Matemática & $2,3 \%$ \\
\hline Tecnología Química & $1,2 \%$ \\
\hline Ing. Minas & $4,8 \%$ \\
\hline Ing. Agronómica & 4,4 \\
\hline Ing. Pecuaria & 3,7 \\
\hline Ing. Biotecnológica & 3,5 \\
\hline TOTAL & $\mathbf{1 0 0}$ \\
\hline
\end{tabular}

Fuente: División de sistemas, 2009

A través de la figura 6 se puede observar que el perfil del bachiller que ingresó a primer semestre de la universidad proviene de bachillerato Académico o Técnico

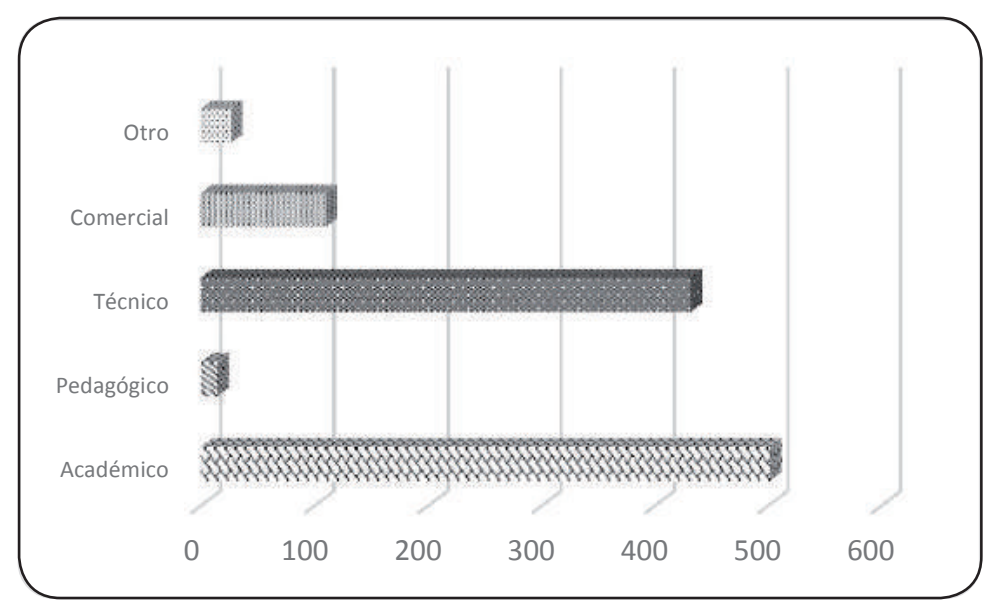

Figura 6 Modalidad de Colegio que proviene

Fuente: autores

42

\subsection{Información académica}

A continuación se presentan los porcentajes de acierto, desacierto y no respuesta en cada una de las preguntas del test las cuales fueron clasificados en pensamientos tal como lo referencia el Decreto 1860 para la educación básica y media (Tabla IV). 
Tabla IV. Porcentajes de Respuestas al test

\begin{tabular}{|c|c|c|c|c|}
\hline$\#$ & Pensamiento & \% Correcto & $\%$ Incorrecto & \% No Responde \\
\hline 1 & Numérico & 82,1 & 15,8 & 2,1 \\
\hline 2 & Numérico & 63,6 & 35,8 & 0,6 \\
\hline 3 & Numérico & 48,4 & 50,2 & 1,4 \\
\hline 4 & Numérico & 28,3 & 70,9 & 0,8 \\
\hline 5 & Variacional & 26,0 & 71,5 & 2,5 \\
\hline 6 & Variacional & 7,3 & 92,4 & 0,3 \\
\hline 7 & Variacional & 36,3 & 63,3 & 0,4 \\
\hline 8 & Variacional & 49,3 & 50,3 & 0,4 \\
\hline 9 & Variacional & 75,6 & 24,0 & 0,4 \\
\hline 10 & Variacional & 19,6 & 78,3 & 2,1 \\
\hline 11 & Variacional & 36,4 & 62,0 & 1,6 \\
\hline 12 & Variacional & 21,0 & 74,9 & 4,1 \\
\hline 13 & Variacional & 27,4 & 71,3 & 1,3 \\
\hline 14 & Variacional & 14,3 & 83,8 & 1,9 \\
\hline 15 & Numérico & 32,8 & 65,8 & 1,4 \\
\hline 16 & Numérico & 19,9 & 78,5 & 1,6 \\
\hline 17 & Numérico & 15,8 & 81,7 & 2,5 \\
\hline 18 & Espacial & 21,3 & 74,3 & 4,4 \\
\hline 19 & Espacial & 46,1 & 52,0 & 1,9 \\
\hline 20 & Espacial & 19,0 & 75,1 & 5,9 \\
\hline 21 & Espacial & 27,3 & 65,2 & 7,5 \\
\hline 22 & Espacial & 44,9 & 54,2 & 0,9 \\
\hline 23 & Espacial & 25,1 & 73,0 & 1,9 \\
\hline 24 & Espacial & 59,8 & 38,3 & 1,9 \\
\hline 25 & Espacial & 35,1 & 52,9 & 2,0 \\
\hline 26 & Espacial y Variacional & 22,5 & 74,2 & 3,3 \\
\hline 27 & Espacial y Variacional & 12,1 & 83,9 & 4,0 \\
\hline 28 & Espacial y Variacional & 24,4 & 41,5 & 34,1 \\
\hline 29 & Espacial y Variacional & 24,1 & 39,3 & 36,6 \\
\hline 30 & Variacional (Problema) & 47,0 & 36,1 & 16,9 \\
\hline 31 & Variacional (Problema) & 47,9 & 16,3 & 35,9 \\
\hline 32 & Espacial (Problema) & 0,9 & 20,8 & 78,4 \\
\hline
\end{tabular}

Enero-Diciembre 2016

ISSN 1794-8231

E-ISSN $2462-8794$

Fuente: autores

Con la información de la tabla IV se pueden calcular los promedios en cada opción de respuesta considerando el tipo de situación propuesta (ejercicio o problema). Los resultados fueron (tabla V):

Tabla V. Promedios en cada opción de respuesta considerando el tipo de situación propuesta

\begin{tabular}{|c|c|c|c|}
\hline Situación propuesta & \% Correcto & \% Incorrecto & \% No Responde \\
\hline Ejercicio & 33,3 & 61,9 & 4,5 \\
\hline Problema & 31,9 & 24,4 & 43,7 \\
\hline
\end{tabular}

Fuente: Autor

Los resultados anteriores, muestran deficiencias en el manejo de preconceptos básicos para iniciar cursos de cálculo. De otra parte, es importante señalar que al observar comportamientos frente a la solución de situaciones problema existe una alta no respuesta (Los problemas corresponden a enunciados de grado 3 de educación básica primaria). 
No. 1

Enero-Diciembre 2016 ISSN 1794-8231

Al observar los resultados por programas, según se muestra en la tabla VI, se resalta el porcentaje de acierto $60 \%$ de respuestas correctas a ejercicios realizados por estudiantes de Ingeniería de Minas y el bajo número de ejercicios resueltos correctamente en estudiantes de Licenciaturas.

Tabla VI. Porcentajes de Respuestas de acuerdo al programa académico

\begin{tabular}{|l|c|c|c|}
\hline PROGRAMA ACADÉMICO & \% CORRECTO & \% INCORRECTO & \% NO RESPONDE \\
\hline Ingeniería Agronómica & 40,8 & 50,8 & 8,4 \\
\hline Ingeniería Mecánica & 57,4 & 35,7 & 6,9 \\
\hline Ingeniería Civil & 32,3 & 65,6 & 2,1 \\
\hline Ingeniería de Sistemas & 55,0 & 38,7 & 6,3 \\
\hline Ingeniería Electrónica & 20,8 & 73,4 & 5,8 \\
\hline Ingeniería Electromecánica & 40,2 & 54,7 & 5,1 \\
\hline Ingeniería Industrial & 42,7 & 51,7 & 5,6 \\
\hline Ingeniería de Minas & 60,0 & 22,2 & 7,8 \\
\hline Ingeniería Agronómica & 27,0 & 63,5 & 9,5 \\
\hline Ingeniería Pecuaria & 26,9 & 61,8 & 11,3 \\
\hline Ingeniería Biotecnológica & 26,9 & 61,8 & 8,1 \\
\hline Administración de Empresas & 29,2 & 62,7 & 8,0 \\
\hline Contaduría Pública & 30,9 & 61,1 & 6,2 \\
\hline Tecnología Química & 41,9 & 51,9 & 6,2 \\
\hline Licenciatura Matemática & 15,5 & 78,3 & \\
\hline
\end{tabular}

Fuente: autores

Un $86 \%$ de los estudiantes admitidos que ingresaron para el segundo semestre del 2009 reconocen tener falencias en cuanto al manejo de conceptos básicos de matemáticas. Del porcentaje de estudiantes que manifestaron no estar interesados en el curso, un alto número argumenta el no realizarlo por trabajar en horarios extraclase o falta de herramientas tecnológicas.
Resultados de análisis cualitativo muestra motivación por implementar gráficos inicialmente en el papel y posteriormente construir modelo utilizando software de apoyo o aplicativos gratuitos para verificar resultados de análisis, estudiantes redactan procedimiento de manera correcta y utilizan lenguaje algebraico adecuado. A lo anterior se diseña curso de precálculo dispuesto en [26]

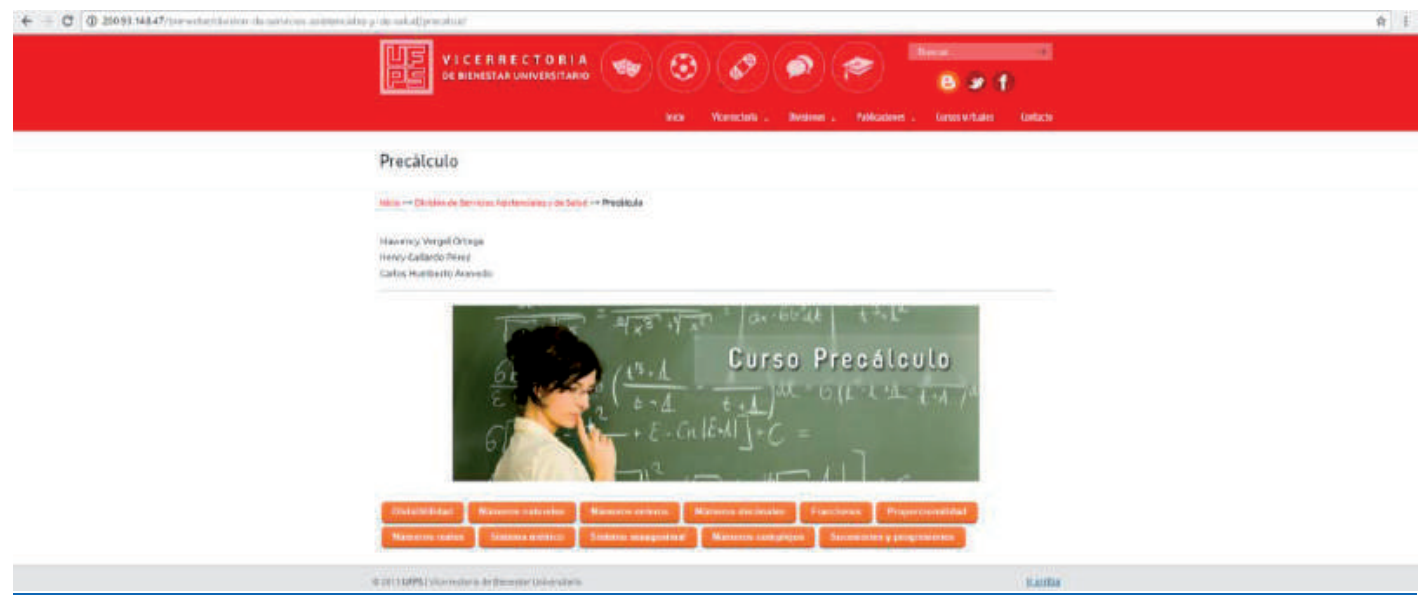

Figura 7 Curso Precalculo Bienestar Universitario Fuente[26] 
Analizando los resultados para el problema P1, se observa que hay diferencias significativas entre el pretest y el postest en ambos grupos $(\operatorname{sig}=0.000)$. Además, hay diferencias significativas según el grupo, experimental o control $(\mathrm{sig}=0.037<0.05)$. Por lo tanto, podemos concluir afirmando que la utilización de curso precálculo favorece el desarrollo de competencias en interpretación, pensamiento variacional y preconceptos matemáticos [27]. El análisis de varianza muestra que hay diferencias significativas entre los resultados del postest y del pretest ( $\mathrm{p}$-valor $0.000<$ 0.05), pero independientemente del grupo $(\operatorname{sig}=0.345)$. Por lo tanto, la respuesta a este problema de investigación es que el uso plataforma virtual utilizando curso precálculo no favorece significativamente el cambio de creencias sobre su enseñanza en estudiantes de primer semestre.

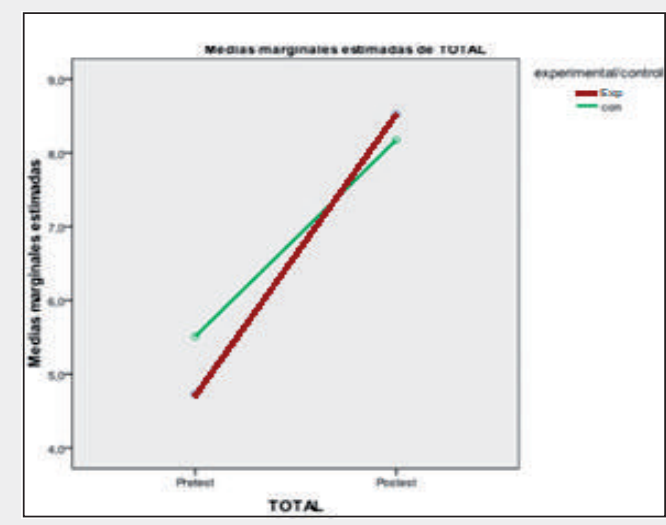

Figura 8. Grafica de análisis de resultados Fuente: autores

\section{Conclusiones}

La utilización de curso precálculo favorece el desarrollo de competencias en interpretación, pensamiento variacional y preconceptos matemáticos.

El uso plataforma virtual utilizando curso precálculo no favorece significativamente el cambio de creencias sobre su enseñanza en estudiantes de primer semestre.
Los docentes deben aprovechar la realidad social que se está viviendo, la utilización o manejo de las TICS [28], están cada día más presente en nuestros estudiantes, se deben tomar todas las ventajas de esta nueva tecnología en el mundo educativo [29] y [30]. Donde el adquirir estas habilidades digitales le ayudan a complementar tareas en el aula. De acuerdo con [31], el planteamiento de actividades enmarcadas en una didáctica pueden promover una mejor comprensión en los conceptos de cálculo dando inicio a una Génesis Instrumental [25] donde el profesor $\mathrm{y}$ estudiantes se familiaricen con los Blogs y los Apps como herramientas de apoyo en la elaboración de los conocimientos.

\section{Referencias}

[1] R. Cantoral, "Hacia una didáctica del Cálculo basada en la cognición. (F. Cordero, R. Farfán, and A. Octak, Edits.) Programa Editorial del Área de Educación Superior del Depto. de Matemática Educativa del CINVESTAV-IPN". Antologías, no.1, pp.1-24, 1994

[2] C. Cuevas y F. Pluvinage, "Cálculo y Tecnología”. El Cálculo y su Enseñanza, vol.1, pp .45-59,2009.

[3] J. Ímaz y L. Moreno, "La génesis y la enseñanza del Cálculo”, Las trampas del rigor. Distrito Federal, México: Trillas. 2010.

[4] A. Aguilar y A. Riestra, "Una introducción algebraica y dinámica al concepto de derivada" (J. Riestra, and C. Cuevas, Edits.) El Cálculo y su Enseñanza, vol.1, pp.1-12, 2009.

[5] C. Cuevas, A. Rodríguez y O. González, "Introducción al concepto de derivada de una función real con apoyo de las tecnologias digitales". (CINVESTAV-
Enero-Diciembre 2016 ISSN 1794-8231 E-ISSN 2462 - 8794 PP: $33-47$ 
I.P.N., Ed.) El Cálculo y su enseñanza, vol. 5, pp. 157-164, 2014.

[6] F. Hitt y S. Dufour, "Un análisis sobre la enseñanza del concepto de derivada en el nivel preuniversitario, del rol de un libro de texto y su posible conexión con el uso de tecnología". En C. Cuevas, and F. Pluvinage, La enseñanza del Cálculo diferencial e integral. Compendio de investigaciones y reflexiones para profesores, formadores e investigadores en Matemática Educativa, pp. 19-42. México D.F: Pearson. 2014.

[7] O. Rincón, "Evaluación de actitudes hacia la incorporación de la Calculadora Voyage 200 en las aplicaciones de las ecuaciones diferenciales de primer orden". Revista Ecomatemático, vol. 2, no.1, pp.21-26, 2011.

[8] T. Dreyfus, "Advanced Mathematical Thimking" P. Nesher and J. Kilpatrick (Ed). Mathematics and Cognition: A Research Synthesis by the Intenational Group for the Psychology of Mathematics Educations. Cambrige University Press. pp. 113-134.1990.

[9] L. Reyes Ruiz, E. Castañeda Carranza, y D. Pabón Castro. "Causas psicosociales de la deserción universitaria". Revista Logos Ciencia \& Tecnología, 4(1), 164-168, 2012. doi: http://dx.doi. org/10.22335/rlct.v4i1.179

46
[10] H. Aebli, Elaborar un curso de acción; Construir una operación; Formar un concepto. En H. Aebli, 12 formas básicas de enseñar. Una didáctica basada en la psicología. 2 ed.,pp. 159233. España: Narcea. 1995.

[11] R. Skemp, Relational understanding and instrumental understanding. Mathematics Teaching, vol. 77, pp. 2026, 1976.
[12] A. Orton, "Students` Understanding of Differentiation". Educational Studies in Mathematics, vol.14, no.3, pp.235250, 1983.

[13] J. Grabiner, "The Changing Concept of Change:The Derivative from Fermat to Weierstrass". Mathematics Magazine, vol. 56, no.4, pp.195-206, 1983.

[14] C.H. Edwards, The Historical Development of the Calculus. SpringerVerlag New York, Inc. USA. 1979

[15] R. Cantoral. Socioepistemología de la variación y el cambio.La enseñanza del Cálculo Diferencial e Integral.Pearson: México. pp.195-216. 2013

[16] R. Hernández, C. Fernández y P. Baptista, Metodología de la investigación (3a ed.). Bogotá, Colombia: McGraw-Hill Interamericana. 2003

[17] P. Verillon, and P. Rabardel, "Cognition and artifacts: A contribution to the study of though in relation to instrumented activity". European Journal of Psychology of Education, vol. 10, no. 1, pp. 77-101, 1995.

[18] M. Vergel Ortega. Test de conocimientos adquiridos durante la educación secundaria. Universidad Francisco de Paula Santander, 2012.

[19] M. Vergel Ortega, H. Duarte, y J. Martínez Lozano. Desarrollo del pensamiento matemático en estudiantes de cálculo integral su relación con la planificación docente. Revista Científica, 3(23), 17-29. 2015. doi:http://dx.doi.org/10.14483/ udistrital.jour.RC.2015.23.a2

[20] M. Vergel, J. Orjuela y J. Martínez, Modelos estimados para el tiempo de permanencia de estudiantes en 
asignaturas de Cálculo en la Universidad Francisco de Paula Santander. En XXVIII Reunión Latinoamericana de Matemática Educativa (Relme). Universidad del Atlántico, Barranquilla, Colombia. 2014

[21] Quédate. [En línea] Disponible en http: HYPERLINK "http://www.ufps.edu. co/ufps/quedate/quedate.php.2013" www.ufps.edu.co/ufps/quedate/ quedate.php.2013

[22] S. Zafra Tristancho, J., Martínez Lozano y M. Vergel Ortega, M, "Indicadores para evaluar la pertinencia social en la oferta académica de programas". Revista Logos Ciencia \& Tecnología, vol. 6, no. 1, pp.165-177, 2014.

[23] J, Martínez, M. Vergel, y L. Zafra. Comportamiento juvenil y competencias prosociales. Bogotá: Ibáñez. 2015.

[24] M. Cárdenas, M. Vergel, O. Rincón, "Reliquidación de matrícula. Caracterización del beneficiario en la Universidad Francisco de Paula Santander". Revista Ecomatemático. Vol. 4, No.1, pp.80-94. 2013

[25] L. Trouche, Managing the complexity of human/machine interactions in computerized learning. International Journal of Computers for Mathematical Learning Environments: guiding students' command process through instrumental orchestrations, 9,pp. 281307. 2004

[26] M. Vergel, H. Gallardo, C. Acevedo. Curso Precálculo Vicerrectoría de Bienestar Universitario. Universidad Francisco de Paula Santander. [On line]. Available: http://200.93.148.47/ bienestar/division-de-servicios- asistenciales-y-de-salud/precalcul/. 2013

[27] C. Acevedo Penaloza, E. Florez Solano, y M. Vergel Ortega. Teoría De Contacto Aplicada Al Mecanismo Leva-Palpador Cilindrico. Bogotá: Editorial Universidad Nacional De Colombia ISBN: 978-958-4492-76-0 v. 1 pags. 58.2012

[28] M. Vergel Ortega, J. Martínez Lozano, y S. Zafra Tristancho, "APPS en el rendimiento académico y autoconcepto de estudiantes de ingeniería" Revista Logos Ciencia \& Tecnología, vol., 6 No.2, pp. 198-208. 2015

[29] A. Atencia Andrade y García Atencia, Corporación Universitaria del Caribe CECAR, Colombia, F. Incorporación de las tic en las metodologías de los docentes de especialización en docencia de cecar. Revista Logos Ciencia \& Tecnología, 5(1), 22-38. 2013. doi:http://dx.doi.org/10.22335/ rlct.v5i1.3

[30] M. Turizo Arzuza. En la búsqueda de nuevas formas de interacción sociodiscursiva en entornos virtuales de aprendizaje: El nuevo rol docente. Revista Logos Ciencia \& Tecnología, 5(2), 263-273, 2014. doi :http://dx.doi. org/10.22335/rlct.v5i2.123

[31] O. Rincón, M. Vergel y S. Ortega, "El Blog como estrategia Didáctica Innovadora en el Aprendizaje del Cálculo". El Cálculo y su Enseñanza, Vol. 6, No. 6.pp.45-70.2014 